\title{
TIPOLOGÍA DE LAS DESCARGAS SOBRE ARENAS DE LA RESERVA BIOLÓGICA DE DOÑANA
}

\author{
José Carlos Muñoz Reinoso \\ Departamento de Biología Vegetal y Ecología. Universidad de Sevilla. Apdo 1.095. 41080-Sevilla \\ Palabras clave: Doñana, ecosistemas de descarga sobre arenas, agua freática, vegetación, hidroquímica, dinámica freática. \\ Key words: Doñana. discharge ecosystems on sandy soils, water table, vegetation, hydrochemistry.
}

\section{ABSTRACT \\ TYPOLOGY OF DISCHARGE AREAS ON SANDY SOILS IN DOÑANA BIOLOGICAL RESERVE}

A typology of discharge areas on sandy soils in Doñana has been carried out based on the vegetation, groundwater hydrochemistry and water tablc regime. Water table (annual and interannual) fluctuations have been related to the connection with different groundwater flow systerns (local, intermediate and regional). Five types of ponds are proposed largely corresponding to previous classifications: permanent ponds, temporary ponds, ephemeral ponds, "fern" ponds and wet slacks. A hypothesis of the desiccation of cphemeral ponds connccted to intermediate flows is considered.

\section{INTRODUCCIÓN}

El acuífero Almonte-Marismas no es sólo un importante factor ecológico en cuanto a su relación con los ecosistemas terrestres (RAMÍREZ DÍAZ 1973, ALLIER et al. 1974), sino que es el factor fundamental para el mantenimiento de los distintos ecosistcrnas acuáticos (lagunas y marismas) por los que Doñana ha recibido un amplio reconocimiento internacional (Rescrva de la Biosfera, zona RAMSAR, ZEPA, Diploma Europeo, Patrimonio de la Humanidad).

Los ecosistemas acuáticos de la Reserva Biológica de Doñana (R.B.D.) dependen de la cantidad y calidad del agua de descarga del acuífero Almonte-Marismas. Hasta hace relativamente poco, se manejaba el modelo de un acuífero libre que se convierte en confinado bajo las marismas (IGME 1983). CUSTODIO et al. (1992) y SALVANY y CUSTODIO (1995) proponen la existencia de un nivel profundo más transmisivo, recubierto por un medio arenoso, a veces con notables intercalaciones arcilloarenosas, de notable menor permeabilidad, y que es por donde se produce la recarga así como las descargas naturales a arroyos, costa. lagunas y vegetación.

Se interpreta pues, como un acuífero semiconfinado por un acuitardo (en sentido relativo) en el área considerada como libre, que pasaría a cautivo estricto bajo la marisma, a través de un tránsito en el que hay un cambio sedimentológico por flexión (CUSTODIO et al. 1992).
Las aportaciones de agua, tanto de lluvia como superficiales, y en menor grado las subterráneas, dependen de la variabilidad de la precipitación. En nuestra área de estudio, tanto la distribución anual de las precipitaciones como la interanual son muy variables, condicionando fuertemente fenómenos hidrológicos como la recarga del acuífero y la escorrentía superficial. Esto unido a la heterogeneidad espacial en cuanto a la presencia de intercalaciones arcillosas, sedimentos lagunares, paleosuelos enterrados, crean comportamientos hidrológicos complejos, de forma que una misma zona puede funcionar temporalmente como área de recarga o como área de descarga (CUSTODIO 1995).

La adaptación a esta variabilidad espacial y temporal viene reflejada por las características de la distribución de las biocenosis de las zonas húmedas, particularmente de la vegetación. Por ello, la zonación característica de las especies Sreatófitas y helófitas ha sido frecuentemente utilizada para la tipificación de aguas en estepas y zonas áridas (GONZÁLEZ BERNÁLDEZ 1988).

La rnayoría de los humedalcs de zonas áridas, tales como lagunas y lagos, se encuentran en zonas de descarga de sistemas de flujo de aguas subterráneas los cuales controlan su régimen hídrico, hidroquímica y biocenosis, indicando el grado de conexión entre las depresiones y los flujos de aguas subterráneas (GONZÁLEZ BERNÁLDEZ 1988), pudiendo ser usados para su tipificación (GONZÁLEZ BERNÁLDEZ et al. 1988). 
La tipificación de los humedales es una contribución a los procesos de valoración y manejo racional de los recursos que los humedales representan (GONZÁLEZ BERNÁLDEZ 1988), así como de su vulnerabilidad hidrológica (GILVEAR \& McINNES 1995). Aunque originalmente el interés recaía sobre los organismos más conspícuos (aves y vegetación), la necesidad por la conservación se ha extendidoal sistema completo, incluyendo aspectos biogeoquímicos, geomorfológicos e hidrológicos que caracterizan los principales complejos ambientales de un territorio.

El objetivo de este trabajo es tipificar los ecosistemas de descarga sobre arenas de la Reserva Biológica de Doñana en base a la hidroquímica del agua subterránea, su vegelación y, fundamentalmente, al cornportaniiento hidrológico dc estos ecosistemas.

En 1987, BORJA y DÍAZ DEL OLMO propusieron una tipificación de los coinplejos húmedos del Abalario atendiendo a la inorfogénesis lagunar, clasificándolos en complejos lagunares sobre cubetas deflactadas (de morfología elongada bilobulada o redondeada) y lagunas procedentes de la obturación de drenajes, relacionándolos con toposecuencias de vegetación según su gradiente de humedad y trofismo. Atendiendo a los procesos morfocdáficos consideran la alimentación por mantos freáticos que originan horizontes de tipo gley, y la alimentación por drenajes laterales, relacionados con aguas superficiales y que originan horizontes tipo pseudogley. Aunque se trata de un área próxima a la R.B.D., no se abordan las características del agua freática y la vegetación se trata de una forma inuy general.

GARCíA NOVO et al. (1991) han propuesto una clasificación de las lagunas de Doñana basada en datos fisicoquímicos, de salinidad y eutrofia de las aguas superficiales, y en la relación de éstas con las descargas del acuífero. El análisis incluye un conjunto muy hcterogénco dc cuerpos de agua, en circunstancias de homogeneización de aguas, y que tiene en cuenta lundamentalmentc la limnología de las aguas superficiales (eutrofia). Se distinguen las lagunas peridunares, permanentes y temporales, frente a lagunas efímeras que pueden mostrar distinto grado de eutrofia según el período de inundación y las características del sustrato de la cubeta.

Posteriormente. BRAVO y MONTES (1993) presentan un inventario exhaustivo de las formaciones palustres del manto eólico del Parque Nacional de Doñana, considerando sectores ambientales, régimen hídrico, topografía y datos morfométricos. Entre estos últimos se han detectado iinportantes errores en cuanto a la superficie de las lagunas. Como aporte de interés están la consideración de los sectores ambientales o subunidadcs geomorfológicas (flecha litoral, manto arrasado, frentes dunares móviles, corrales, contacto naves-manto arrasado, contacto arenas móviles-manto arrasado y contacto manto arrasado-marisma) y el régimen hídrico (estacional, semipermanente, permanente, influencia mareal).

\section{MATERIAL Y MÉTODOS}

Se han retornado las matrices de datos de vegetación y fisicoquímicos utilisadas en un trabajo anterior (MUÑOZ REINOSO 1995) eliminando las muestras con un carácter marcadamcnte terrestre y aquellas con altos contenidos iónicos. En su lugar se han incluido tres nuevos puntos: las lagunas de Santa Olalla (SOL) y Zahillo (ZAH), y un segundo punto en el Corral Largo (CL2). Como datos de hidroquímica del agua freática de estos puntos se han utilizado los publicados en el trabajo de LÓPEZ et al. (1994) (Tabla 1).

Tabla 1. Características fisicoquímicas niedias del agua freática y variables "hidrodinámicas" en los puntos muestreados.

Table I. Mean physical and chemical characteristics of the groundwater and the "liydrodinarnic" variables of the sampling points.

Abreviatura/Abbreviations: $\mathrm{CA}$ : resevn alcalina-alkalinity; $\mathrm{Cl}$ : cloruro:chloride Con: conductividad-electrical conductivity; Na: sodio-sodium; PNF: profundidad del nivel freático-water table depth; FLU: fluctuación anual-water tnble annual fluctuation: INT': fliictuación interanual-water table interannual fluctuation.

\begin{tabular}{lcccccccc}
\hline & $\mathrm{pH}$ & $\begin{array}{c}\text { Cond } \\
(\mu \mathrm{S} / \mathrm{cm})\end{array}$ & $\begin{array}{c}\mathrm{Na} \\
(\mathrm{meq} / \mathrm{l})\end{array}$ & $\begin{array}{c}\mathrm{Cl} \\
(\mathrm{meq} / \mathrm{l})\end{array}$ & $\begin{array}{c}\mathrm{CA} \\
(\mathrm{meq} / \mathrm{l})\end{array}$ & $\begin{array}{c}\mathrm{PNF} \\
(\mathrm{m})\end{array}$ & $\begin{array}{c}\text { FLU } \\
(\mathrm{m})\end{array}$ & $\begin{array}{c}\text { INT } \\
(\mathrm{m})\end{array}$ \\
\hline OJILLO & 68 & 204 & 102 & 254 & 1.51 & 135 & 0.05 & 113 \\
ACEBUCHE & 68 & 202 & 079 & 200 & 087 & 166 & 009 & 110 \\
TARAJE & 69 & 318 & 121 & 383 & 3.32 & 088 & 084 & 059 \\
DULCE & 71 & 565 & 288 & 287 & 390 & 125 & 074 & 028 \\
C LARGO & 67 & 145 & 058 & 287 & 090 & $\mathbf{1} 79$ & 020 & 049 \\
ALGAIDA & 73 & 478 & 106 & 326 & 115 & 097 & 071 & 024 \\
JABATA & 67 & 228 & 106 & 512 & 067 & $\mathbf{1 3 2}$ & 075 & 011 \\
M PAVON & 66 & $\mathbf{1 6 8}$ & 045 & 456 & 083 & $\mathbf{1 0 7}$ & 065 & 034 \\
S OLALLA & 75 & 556 & 543 & 734 & 1406 & $\mathbf{1} 14$ & 055 & 033 \\
ZAHLLLO & 83 & 499 & 3.04 & 612 & 308 & $\mathbf{1 1 3}$ & 064 & 084 \\
C LARGO-2 & 65 & 206 & 183 & 3.26 & 078 & 148 & 018 & 035 \\
\hline
\end{tabular}

Las áreas de descarga estudiadas (Fig. 1) se encuentran localizadas en las zonas de transición entre las distintas unidades ambientales y geoinorfológicas de la Reserva Biológica de Doñana (ALLIER et al. 1974): arenas estabilizadas, dunas móviles y marisma. Dentro de las arenas estabilizadas se distinguen las Naves (al Oeste y constituyendo el área topográficamente más elevada de la R.B.D.) y el Manto Arrasado (al Estc y mostrando una morfología dunar más destruida). Más al Estc, la transición entre las arenas estabilizadas y las arcillas de la marisma recibe localmente el nombre de Vera. En las dunas móviles aparecen los corrales o valles interdunares cubiertos por vegetación.

Se han incluido dos nuevas variables que se relacionan con el funcionamiento hidrológico. La priincra variable incluida ha 
sido la fluctuación anual (FLU), considerada como la media de las diferencias de las profundidades mínima y máxima a la que sc encontraban los niveles freáticos en los distintos puntos para los años hidrológicos 93-94 y 94-95, y que representa la respuesta a las entradas (precipitaciones y descargas subterráneas fundamentalmente). Sc ha calculado corno la diferencia entre la profundidad mínima, que suele alcanzarse

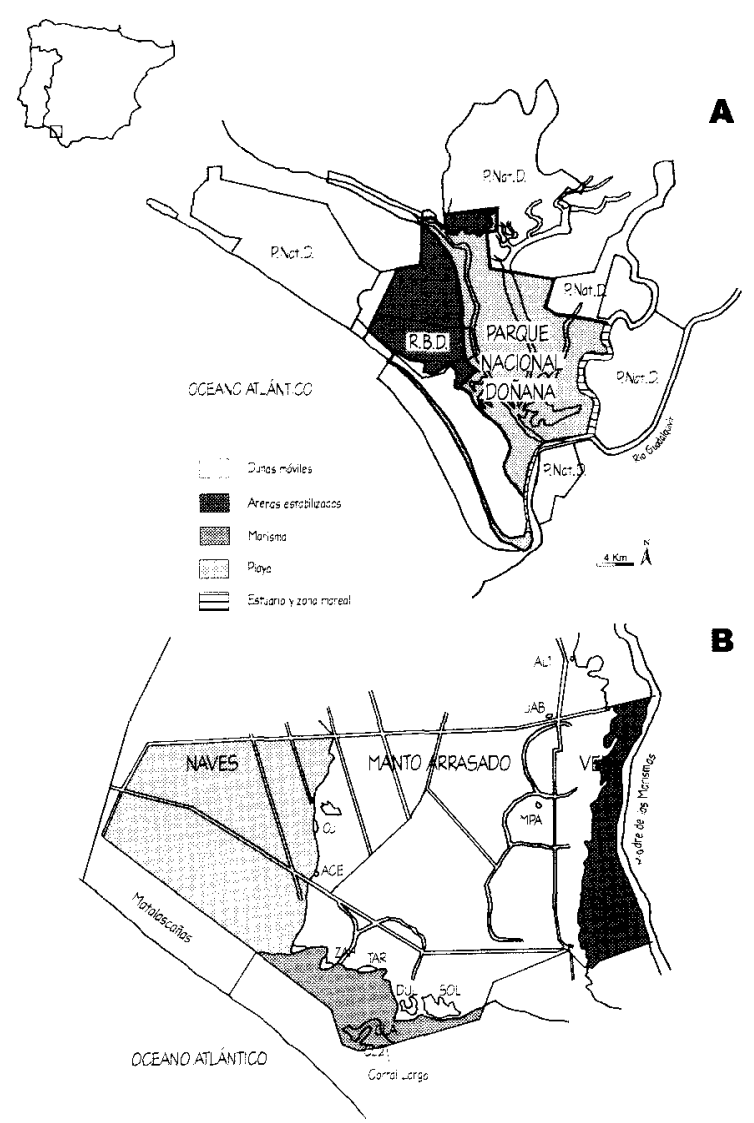

Figura 1. A) Parque Nacional y Parque Natural de Doñana. Localización de la Reseva Biológica de Doñaiia (R.R.D.) B) Reserva Biológica de Doñana. Localización de los puntos de muestren.

Figure 1. A) National and Natural Parks of Donana. Location of Doñaiia Biological Reserve (R.B.D.) B) Doñana Biological Reserve. Location of sampling points.

Abreviaturas/Abbreviations: Ojillo (OJI), Acebuche (ACE), Taraje (TAR), Zahillo (ZAH), Corral Largo (CLA), Corral Largo 2 (CL"), Dulce (DUL). Santa Olalla (SOL). Martín Pavón (MPA), La Jabata (JAB), La Algaida (ALI).

cntre febrero y marzo (antes del aumento de la evapotranspiración), salvo en el caso de importantes precipitaciones primaverales, y la profundidad ináxima registrada antcs de iniciarse el ascenso de los niveles, al final del año hidrológico anterior y que, en algunos casos, se extiende hasta el inicio de lluvias importantes.
La segunda variable ha sido la fluctuación interanual (INT). Ésta se ha calculado como la diferencia existente entre los mínimos de los años hidrológicos 1991-92 y 1994-95, que varía entrc los $11 \mathrm{~cm}$ en La Jabata y los 113 en Ojillo, y da idea del aporte dc agua subterránea durante este período de sequía. Debido a que el piezómetro de La Jabata ha sido repctidamentc inutilizad», se le ha asignado el valor de un punto próximo (Helechal del pinar de Martinazo).

Para el Análisis Canónico dc Correspondencias (TER BRAAK 1991) se han eliminado las variables $\mathrm{pH}$, potasio, magncsio y calcio, y las especies que aparecían en una sola muestra. Finalmente se realizó el análisis con 11 muestras, 14 especies y 7 variables.

Por otro lado, se ha realizado un análisis de clasificación de tipo aglomerativo según las variables "hidrodiriáinicas" (profundidad del nivel freático, fluctuación y descenso intcranual) de los 11 puntos de mucstreo, utilizando el porcentaje de similaridad como algoritmo. Previamente las variables han sido transformadas logarítinicamentc.

En tercer lugar, se han recopilado los registros piezométricos realizados a lo largo de varios años en distintos puntos de descarga sobre las arenas de Doñana (arenas móviles, arenas estabilizadas y zonas de transición entrc éstas y entre éstas y las marismas).

\section{RESULTADOS}

\section{Análisis Canónico de Correspondencias}

La Tabla 2 y la Fig. 2 rcsumcn el resultadodel Análisis Canónico de Correspondencias. Las correlaciones de las especies con los ejcs, rcsultado de la combinación lineal de las variables ambientales, son muy altas, superiores a 0.95 para los dos primeros ejes.

Tabla 2. Resultados del análisis Canónico de Correspondencias. En itálicas se destacan las correlaciones más altas entre las variables ambientales y los ejes. Table 2. Sunirnary results of the Canonical Correspondence Analysis. In italics tlie higher correlation values between environmental variables and axis.

Variables ambientales1 Environmental variables: CA: reserva alcalina-alkalinity; CI: cloruro-chloride; Con: conductividad-electrical conductivity; Na: sodiosodium; PNF: profundidad del nivel freático-water table depth; FLU: fluctuación anual-water table annual tluctiintión: INT: fluctuación interanual-water table interannual fliictiiation.

\begin{tabular}{|c|c|c|c|c|}
\hline & EJE I & EJE II & EJE III & EJE IV \\
\hline Autovalores & 0885 & 0565 & 0287 & 0.125 \\
\hline $\begin{array}{l}\text { Correlaciones especies-variables } \\
\text { ambientales }\end{array}$ & 0.983 & 0958 & 0.923 & 0.907 \\
\hline Varianza explicada (especies) & 37.3 & 23.7 & 12.1 & 5.3 \\
\hline $\begin{array}{l}\text { Varianza explicada (especies-v ambientales) } \\
\text { Correlaciones variables ambientales-ejes }\end{array}$ & 44.4 & 28.4 & 14.4 & 6.2 \\
\hline Con & -0.34 & $-6.7 t$ & -0.22 & -0.22 \\
\hline $\mathrm{Na}$ & -0.67 & -0.46 & -0.29 & -0.06 \\
\hline $\mathrm{Cl}$ & 0.11 & -0.58 & 0.24 & -0.06 \\
\hline $\mathrm{CA}$ & -0.64 & -0.57 & -0.10 & -0.12 \\
\hline PNF & -0.22 & 0.72 & -0.24 & -0.34 \\
\hline FLU & 0.23 & -0.85 & -0.14 & 0.25 \\
\hline INT & -0.40 & 0.41 & 0.70 & -0.13 \\
\hline
\end{tabular}


56

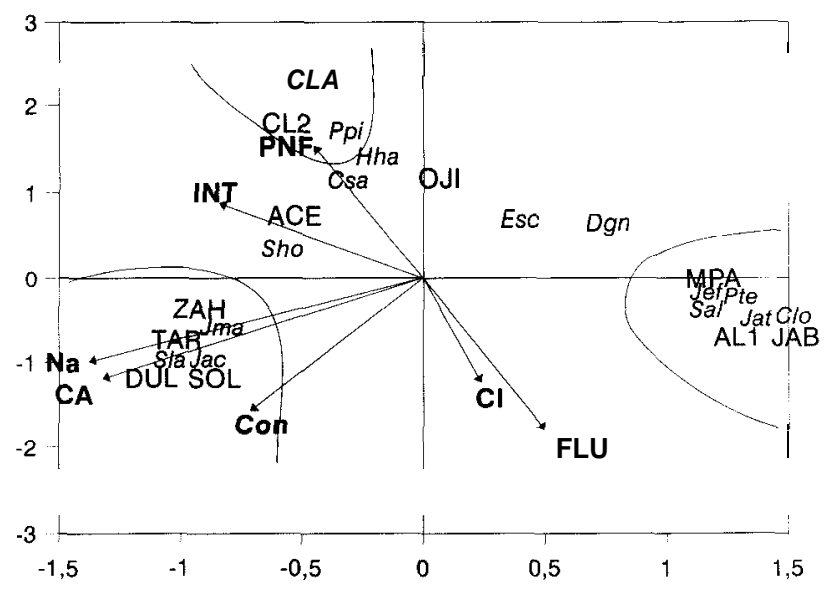

1.ịgura L. Diagrama de ordenación del Análisis Canónico de Correspondencias. Figure 2. Canonical Correspondence Analysis ordinntion diagram.

Especies/Species: Csa: Cistus salvifolius; Clo: Cyperus Longus, Dgn: Daphe gnidiunt, Esc: Erica scoparia; Hha: Halimium halimifoliam; Jat: Juncus acutiflorus; Jac: J. acutus; Jef: J. effusus; Jma: J. Maritinus: Ppi: Pinus pmea: Pte: Pteridium aquilinum, Sal: Salir atrocinerea, Sci: Scirpus holoschoenus, Sla: Scirpus lacustris.

Variables ambientales/ Environmental variables: CA: reserva alcalina-alkalinity; CI: cloruro-chloride: Con: conductividad-electrical conductivity; Na: sodiosodium: PNF: profundidad del nivel freńtico-water table depth; FLU: tluctiiación anual-water tnble annual fluctuación: INT: tliictuación interanual-water tnble interannual fluctuation.

El primer eje absorbe un $44.4 \%$ de la varianza y está correlacionado negativamente con la reserva alcalina (-0.64) y el contenido en sodio (-0.67). Separa por un lado las pequeñas lagunas de la Vera (La Jabata, La Algaida y Martín Pavón) con aguas poco mincralizadas, caracterizadas por una banda de helechos. con helófitos glicófitos (J.effusus, I. acutiflorus y Cyperus longus), y la presencia de Salix atrocinerea. Los restantes puntos se separan a lo largo del segundo eje

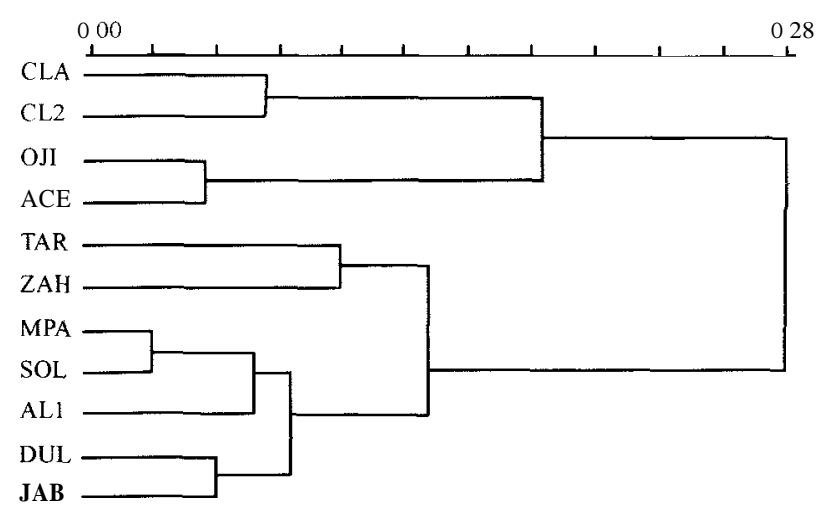

Figura 3. Análisis de clasificación de los puntos de muestreo según las variables "hidrodinámicas", Abreviatiiras en fig. 1 .

Figure 3. Cluster analysis of samplig points based on "hydrodynamic" variables. Abbreviations in fig. 1
El segundo eje, que absorbe un $28.4 \%$ de la varianza, está correlacionado positivamente con la profundidad del nivel freático $(0.72)$ y negativamente con la conductividad $(-0.74)$ y las fluctuaciones anuales (-0.85). En general, se mantiene el gradiente de mineralización, tomando importancia las variables hidrodinárnicas. En la parte negativa aparecen las lagunas peridunares (Zahillo. Tarajc, Dulce y Santa Olalla), con aguas freáticas más mincralizadas, mayores respuestas a la recarga y menores descensos interanuales, con una vegetación compuesta por Juncus maritimus, J. acutus y Scirpus lacustris.

En la parte positiva aparecen los puntos localizados en el Corral Largo, con nivel freático más profundo, aguas poco mineralizadas, pequeñas respuestas anuales y descensos interanuales intermedios.

\section{Clasificación según las características freáticas}

La Fig. 3 muestra el resultado del análisis de clasificación. Puederi distinguirse dos grupos, dentro de los cuales pueden distinguirse a su vez otros dos. En el primer grupo aparecen los puntos del Corral Largo y las lagunas de la transición NavesManto Arrasado (Ojillo y Acebuche de Matalascañas), y por otro lado están las lagunas peridunarcs (Santa Olalla, Dulce. Zahillo y Taraje) y las de la Vera (La Jabata, Martín Pavón y La Algaida).

En el primer grupo se encuentran los puntos de muestreo con nivel freático más profundo, menor respuesta a las entradas y mayores descensos interanuales. Se distinguen claramente los puntos del Corral Largo frente a Ojillo y Acebuche debido a las diferencias en cuanto al descenso interanual, con rnedias de 0.42 y 1.12 in respectivamente.

Las lagunas del segundo grupo presentan aguas con mayores respuestas a la recarga y niveles freáticos más superficiales. Entre ellas, Taraje y Zahillo presentan mayores descensos interanuales, mientras que Dulce, Santa Olalla y las lagunas de la Vera presentan menor descenso interanual en conjunto.

\section{Seguimiento de los niveles freáticos}

Sc han agrupado los puntos de inucstreo de acuerdo al análisis de clasificación y a su localización, representándose la variación mensual de los niveles en los distintos puntos frente a las precipitaciones registradas en el Corral Largo (Fig. 4-7). En la Fig. 7 se muestran juntos Ojillo, Acebuche y un piezómetro del Corral Largo. De esta forma se observan más claramente las diferencias entre ellos, no considerándose necesaria la inclusión del segundo punto del Corral Largo ya que su evolución es similar. 


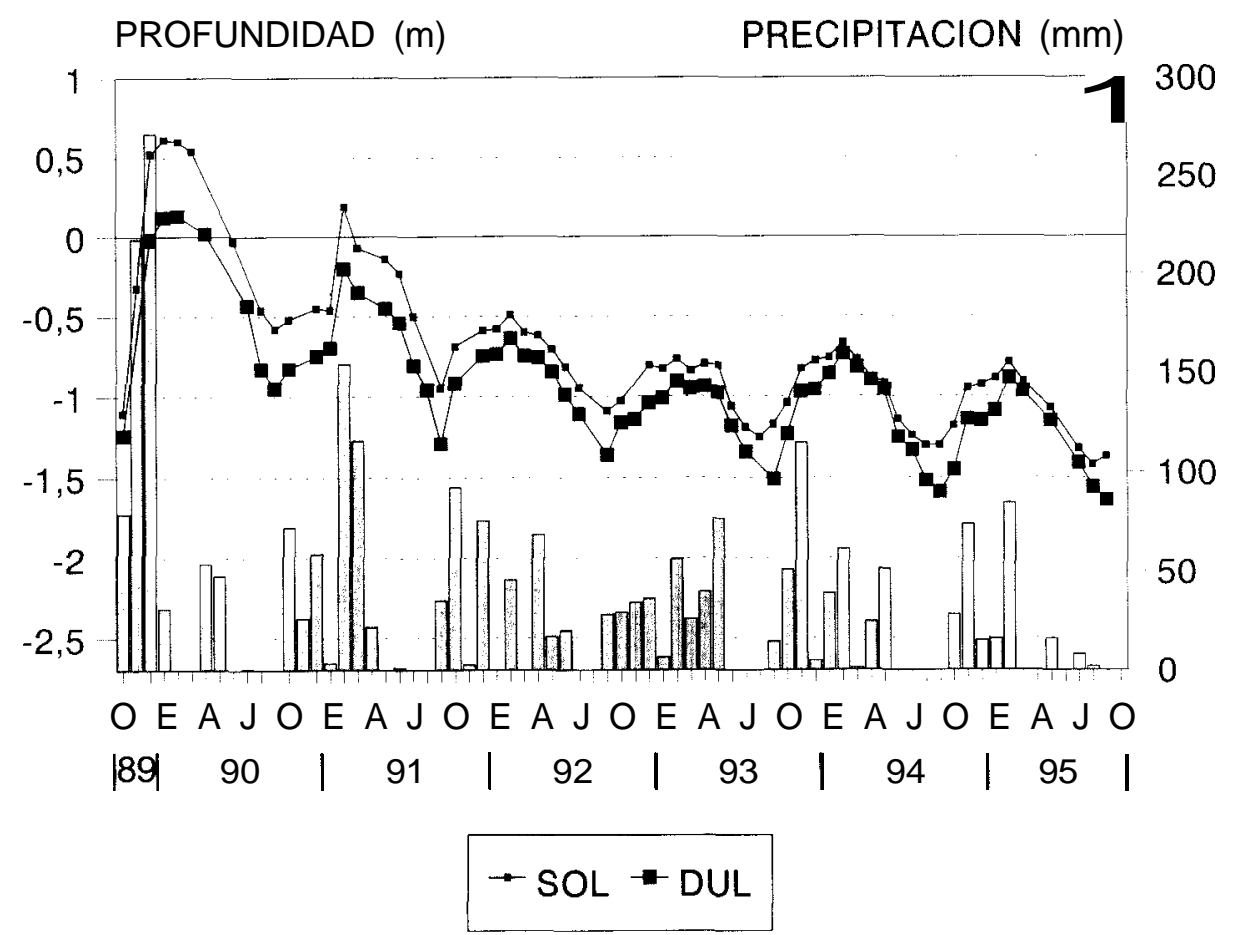

Figura 4. Fluctuación de los niveles freáticos frente a la precipitación en las lagunas peridunares permanentes, Dulce (DUL) y Santa Olalla (SOL).

Figure $\mathbf{J}$. Water table fluctuations versus precipitation in tlie permanent peridune ponds, Dulce (DUI) and Santa Olalla (SOL).

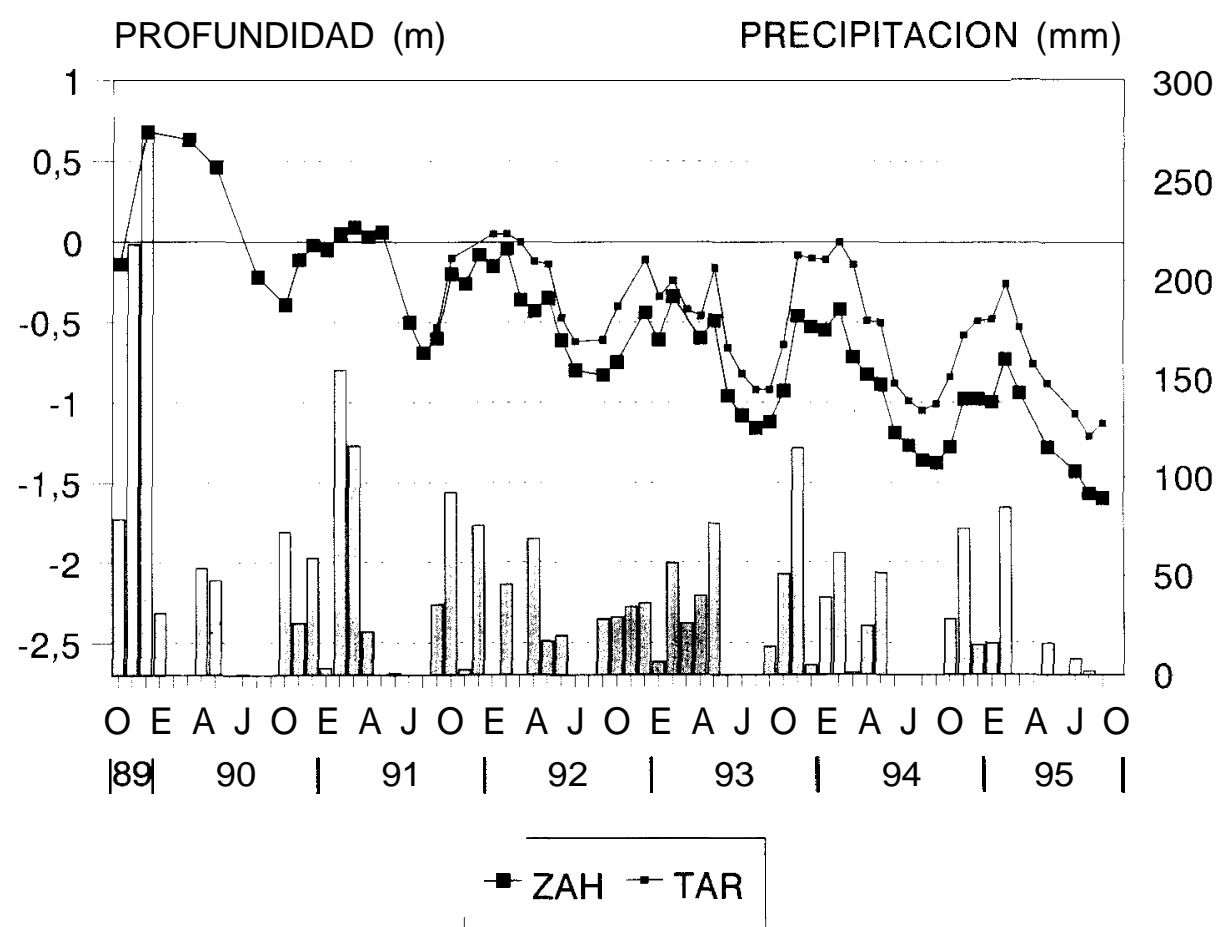

Figura 5. Fluctuación de los niveles freáticos frente a la precipitación en las lagunas peridunares temporales, Zahillo (ZAH)) y Taraje (TAR).

Figure 5. Water table fluctuations versus precipitation in the temporary peridune ponds, Zahillo (ZAH) and Taraje (TAR). 


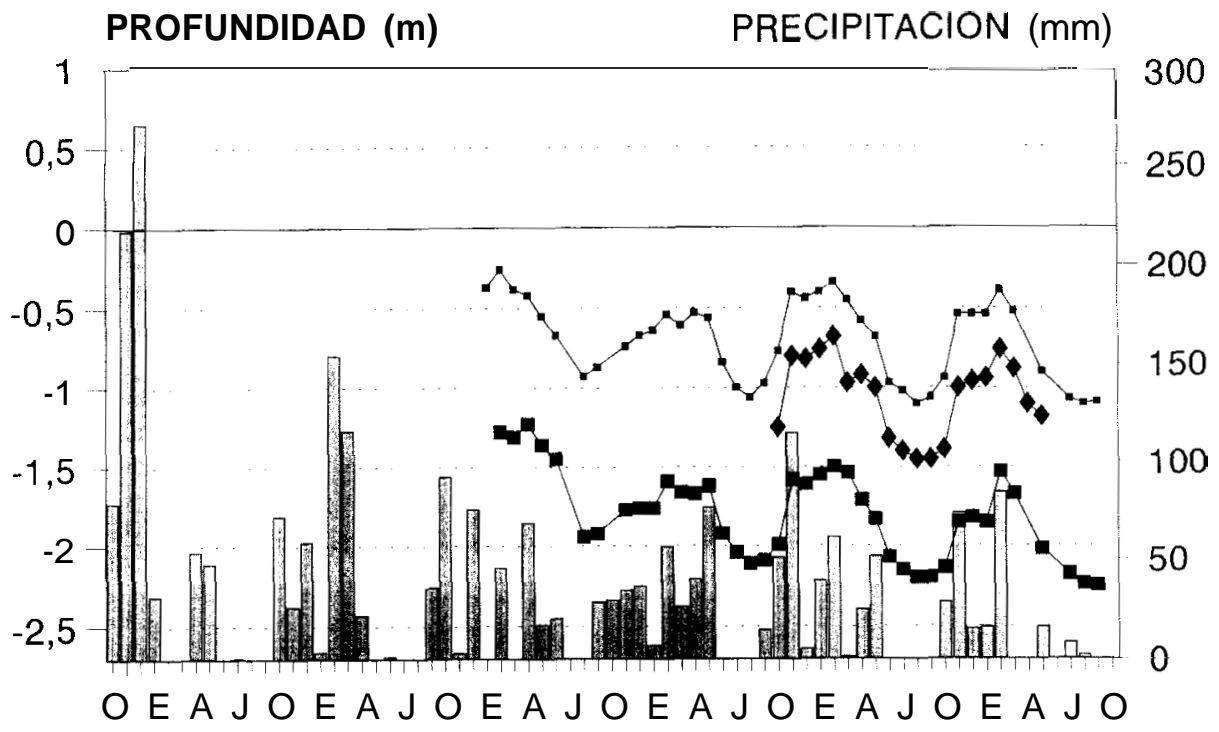

\begin{tabular}{lllllllllllll|}
$\mid 89$ & 90 & $\mid$ & 91 & $\mid$ & 92 & $\mid$ & 93 & $\mid$ & 94 & 95 &
\end{tabular}

$$
\rightarrow \mathrm{MPA} \rightarrow \mathrm{AL1} \bullet \mathrm{JAB}
$$

Figure 6. Fuctuación de los niveles freáticos frente a la precipitación en los helechales, Martín Pavón (MPA), La Jabata (JAB) y La Algaida (AL, I).

Figure 6. Water table fluctuations versus precipitation in the fern ponds, Martín Pavón (MPA), La Jabati (JAB) and La Algaida (ALI).

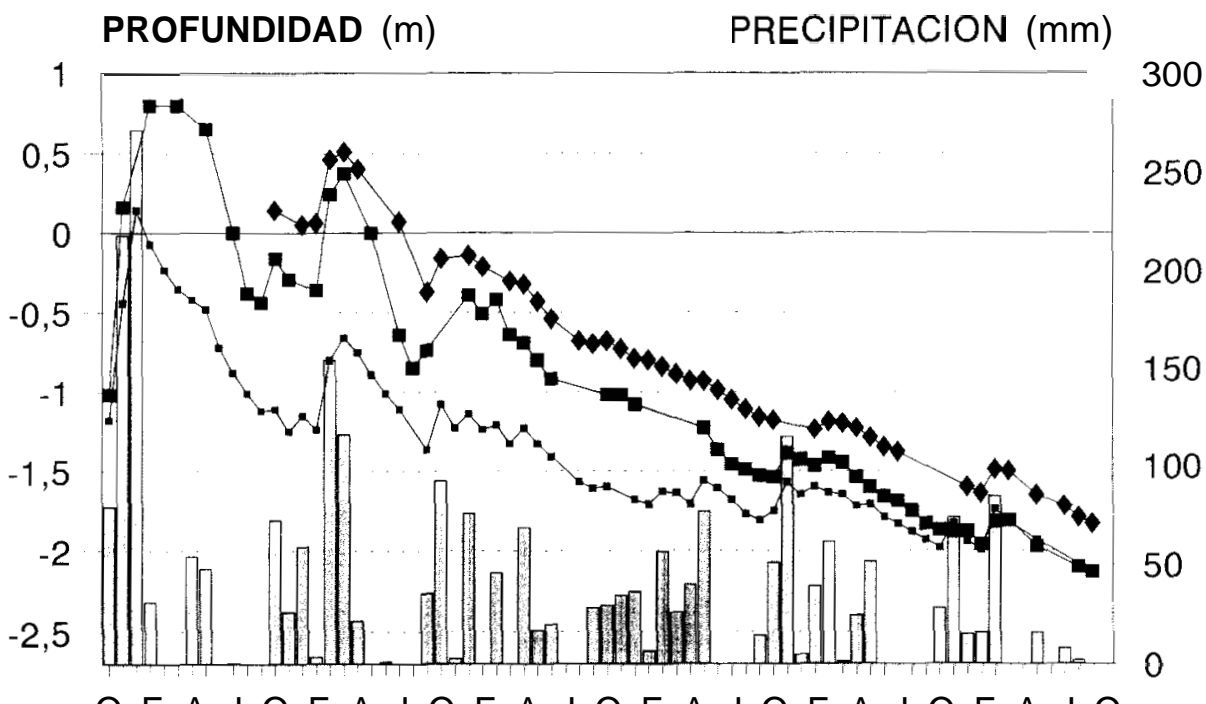

O E A J O E A J O E A J O E A J O E A J O E A J O

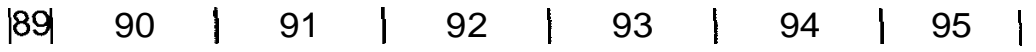

$$
\rightarrow \mathrm{CLA} \rightarrow \mathrm{ACE} \rightarrow \mathrm{OJI}
$$

Figura 7. Fluctuación de los niveles freáticos frente a la precipitación en el Corral 1 argo (CLA) y en las Jagunas de la ti-aniicióii Naves-Manto Arrasado. Acebuche (ACE) y Ojillo (OJI)

Figure 7. Water table fluctuations versus precipitation in ihe Corral Largo (CLA) and in the ponds of the Naves-Manto Arrasado transition, Acebuche (ACE) and Ojillo (OJI). 
En general, se observa que los niveles se ericuentran más próximos a la superficie hacia finales de Febrero y principios de Marzo, excepto cuando existen fuertes precipitaciones primaverales; en ese caso los máxiinos se localizan hacia finales de Abril o principios de Mayo. Igualmente se observa que precipitaciones escasas relativamente similares producen respuestas más acusadas durante la primera parte del período húmedo (Octubre-Febrero) que durante el resto del año. Esto puede ser debido a que los niveles se encuentran más próximos y a que existen inenores salidas (fundamentalmente por evapotranspiración).

En el primer grupo, formado por las lagunas peridunares permanentes (Fig. 4), se observa un comportamiento cíclico, típico de períodos húmedos y secos, con descensos interanuales pequeños $(0.28$ y $0.33 \mathrm{~m}$ para Dulce y Santa Olalla respectivamente).

Aunque todas las lagunas peridunares presentan importantes fluctuaciones anuales, el descenso iriteranual es más marcado en Zahillo y Taraje (0.84 y 0.59 in respectivamente) que en Dulce y Santa Olalla (Fig. 5).

En los puntos de muestreo de la Vera (Fig. 6), los niveles freáticos muestran un comportamiento cíclico con descensos interanuales poco marcados $(0.11,0.24$ y $0.34 \mathrm{~m}$ para La Jabata. La Algaida y Martín Pavón respectivamente). Las respuestas a la recarga son muy similares a las de las lagunas peridunares, pero los descensos son muy bruscos debido a la alta transpiración de los helechos durante su ciclo de vida.

En el grupo formado por Ojillo, Acebuche y el Corral Largo (Fig. 7), solo se observan nítidarnentc ciclos hacia el principio del período representado. con solo ligeras respuestas a las precipitaciones a partir del año 92-93 y un descenso continuado desde entonces. El descenso intcranual de los mínimos desde el año 90 es de unos $1.70 \mathrm{~m}$ para las dos primcras, mientras que para el Corral Largo es de solo un metro. También puede apreciarse como los mínimos del año natural no se hallan al final del año hidrológico (Septiembre), sino que pueden extenderse hasta las siguientes precipitaciones importantes. Las respuestas a la recarga anual son escasas en todos los casos, especialmente en Ojillo y Acebuche.

\section{DISCUSIÓN}

Los resultados de los análisis de orderiacióri (CCA) y de clasificación parecen indicar la existencia de puntos de inuestreo que presentan aguas con características químicas distintas y coinposiciones florísticas relacionadas, corno se ha inostrado anteriormente (MUÑOZ REINOSO 1995). Esto se vc reforzado por la iritroducción de nuevas variables relativas a su régimen hídrico. Por otro lado. los resultados obtenidos muestran una clara segregación espacial entre los puntos estudiados.

De acuerdo con GONZÁLEZ BERNÁLDEZ (1988), el que exista inulticolinearidad de variables puede indicar que muchas de ellas definen los mismos tipos funcionales. Debido a esto. puede intentarse una clasificación de los ccosistemas acuáticos de la Reserva según su hidroquímica, su vegetación y su comportamiento hidrológico (descargas predominantes).

Entre los puntos inuestreados pueden separarse muy claramente Ojillo (OJI), Acebuche de Matalascañas (ACE) y Corral Largo (CLA, CL2) del resto. Se trata de zonas con aguas poco inineralizadas. con fluctiiaciones de los niveles freáticos marcadas durante los dos primeros años hidrológicos y descensos continuados hasta el final. siendo más patente en los dos primeros puntos. Esta gran fluctuación interanual puede indicar una dependencia de la precipitación, presentando un comportamiento complejo, ya que funcionarían fundamentalmente como áreas de recarga y como áreas de descarga temporalmente (CUSTODIO 1995), aunque este comportamiento puede ser relativamente reciente para las dos primcras.

La distinta composición florística así como las diferencias en el funcionamiento hidrológico y su distinta localización- las dos primeras en la transición entre las Naves y el Manto Arrasado, con vcgctación de monte negro y juncos, y la tercera en las dunas con pinos, especies de monte blanco y Scirpus holoschoenus- permiten establecer diferencias entre ellas.

Los niveles en el Corral Largo (MUÑOZ REINOSO 1997) dependen de la precipitación local, presentando un marcado descenso estival. Se trata cle un valle interdunar con descargas locales procedentes de los frentes y colas dunares, y un nivel freático relativamente próximo dentro de lo que puede considerarse un subsistema (el acuífero dunar) del acuífero Almonte-Marismas.

La presencia de especies higrófitas que han experimentado una fuerte mortandad y pérdida de biomasa fotosintética durante la última sequía, unido al fuerte descenso de niveles parece indicar que Ojillo y Acebuche son áreas de descarga de las Naves -3" sistema eólico de RODRÍGUEZ RAMÍREZ (1996)- sobre el Manto Arrasado -2" sistema cólico- sobre el que cabalga. Cuando se produjo este cabalgamiento de un sistema dunar sobre otro más antiguo, es posible que éste último tuviese un incipiente suelo que retrase la infiltración de la lluvia y permita un almacenamiento del agua que descarga posteriorinente por delante del frente de las Naves, tal como hoy lo hacen las dunas móviles hacia el rosario de lagunas peridunares. Otra posible explicación del desnivel podría ser la presencia de la falla del Guadiamar (SALVANY y CUSTODIO 1995). 
Determinadas causas (sequía, cxtraccioncs próximas y evapotranspiración por masa arbórea) han debido reducir este "almacenamiento" de agua, lo cual ha debido ser la causa de la desecación de las pequeñas lagunas de las Naves existentes en 1971 y de la laguna del Brezo (MUÑOZ REINOS0 1997). Este descenso debe haber sido relativamente reciente y se ha visto acelerado durante la últíma sequía, no pcrrnitiendo la adaptación de las plantas cxistcntes en estos enclaves, en tanto que las que se encuentran adaptadas a zonas cori el freático profundo han soportado mejor la sequía.

Por todo ello, Ojillo y Acebuche se encontrarían cri áreas de descarga de flujos intermedios (descarga de las Naves), funcionando actualmente corno áreas de recarga debido a un descenso generalizado de niveles freáticos en la zona. Este proceso se ve enmascarado por períodos con fuertes precipitaciones que permiten la existencia de este tipo de dcscargas, como los años 8990 y $90-91$ y los recientes $95-96$ y 96-97.

El resto dc los puntos inuestreados, lagunas peridunarcs y helechales, presentan ciclos anuales, existicndo pequeñas diferencias en las fluctuaciones de los niveles así como cn sus aguas y composiciones florísticas. Parece existir una cierta independencia de la precipitación local, lo que mostraría su conexión con el acuífero regional, siendo mayor en unos casos que en otros.

Por un lado estarían las lagunas peridunarcs, Santa Olalla, Dulce, Taraje y Zahillo. Las cuatro presentan una vegetación compuesta fundamentalmente por juncos (Juncus acutus, J. maritimus, S. holoschoenus), y un agua freática caracterizada por los carbonatos y en incnor medida por el sodio. determinado por la interacción con las aguas superficiales que presentan estas características (SERRANO y TOJA 1995).

Tradicionalmente cstas lagunas se han corisidcrado alimentadas fundamentalmente por el acuífero dunar (TORRES et al. 1975. GARCÍA NOVO et al. 1976) debido a su localización pcridunar, aunque está demostrada su conexión con el acuífero regional (VELA 1984, SACKS et al. 1992). La observación directa durante períodos lluviosos ha permitido conocer la existencia de importantes aportes de agua en superficie procedentes tanto de las dunas móviles como de las arcnas estabilizadas. El mapa de isopiezas (IGME 1983) confirma este gradiente hidráulico.

De este inodo, puede considerarse que estas lagunas. localizadas en zonas topográficamente más bajas, reciben aguas de distintas procedencias, predominando las aguas subterráneas sobre las superficiales (escorrentía y precipitación). Eritre las subterráneas, que son las menos cambiantes en el tiempo (CUSTODIO 1995). las procedentes del acuífero dunar son menos constantes ya que dependen de la precipitación local. en tanto que las procedentes del acuífero regional son más constantes. Las diferencias entrc Santa Olalla y Dulce frente a Zahillo y Tarajc se deben a la mayor o menor importancia de las descargas regionales, a su nivel topográfico, así como al balance hídrico del cuerpo de agua. Podría decirse que las lagunas tcmporales y permanentes dependen de descargas regionales, dcscargas intermedias y descargas locales. sicrido más importantes las primeras en la lagunas permanentes (Dulce y Santa Olalla). Esto hace que tanto los niveles corno la composición de sus aguas supcrficialcs sean diferentes (LÓPEZ et al. 1991, SACKS et al. 1992. SERRANO y TOJA 1995).

Las pequeñas lagunas de la Vera muestran ciclos anuales con un escaso descenso de los mínimos a lo largo de la sequía. mostrando sus aguas freáticas unos nivelcs relativamente altos de cloruros. Esto último ha sido explicado como resultado de su proximidad a la marisma (SILJESTROM 1985, MANZANO et al. 1991). Por otro lado, se ha considerado la vera, la zona de transición entre las arenas estabilizadas y las arcillas de la inarisnia, como la zona de descarga del acuífero regional (GONZÁLEZ BERNÁLDEZ et al. 1977a y b, MERINO et al. 1980a y b. LLAMAS 1990). Las manifestaciones hidrológicas superficiales observadas en el árca (taludes rezumantes. lagunas y caños -pequeños cauces estacionales que desembocan en la marisma-) son típicas de áreas de descargas de aguas subterráneas (RODRÍGUEZ ARÉVALO y LLAMAS 1986).

El comportamiento de los riivcles, hasta cierto punto independientes de las precipitaciones locales. con ciclos muy constantes en el tiempo, parecen indicar que se trata de descargas de sistemas de flujos subterráneos regionalcs. Estos flujos suelen descargar aguas muy mineralizadas (GONZÁLEZ BERNÁLDEZ y MONTES 1989), aunque esto no parece ser el caso de las descargas regionales en Doñana, donde el material silíceo reacciona poco con el agua de lluvia (FREEZE \& CHERRY 1979). El cscaso descenso de los niveles puede deberse a la constancia de cstas descargas, a la existencia de niveles impermeables debido a interdigitaciones arcillosas de la marisma (SALVANY 1991) o bien de carácter aliótico debido a la formación de costras férricas (SILJESTRÖM 1985), sicrido cstas últimas detcctadas en varios sitios (La Jabata, Martín Pavón). Las especies cxistentcs. como Salix atrocinerea parecen confirmar la constancia de las descargas, ya que esta especie necesita que la zona saturada esté muy próxima a la superficie y que sufra 
pocas oscilaciones (GONZÁLEZ BERNÁLDEZ y MONTES 1989).

Estas lagunas presentan una vegetación glicófita, formada por Pteridium aquilinum, Juncus effusus, J. acutiflorus, Cyperus longus, Iris pseudacorus, Salix atrocinerea, algunas de las cuales han sido consideradas indiferentes en el acuífero dc Madrid (GONZÁLEZ BERNÁLDEZ y MONTES 1989). Los helechales (Mastín Pavón, La Jabata, La Algaida) son pequeñas áreas surgentes que se localizan en dos líneas paralelas próximas al borde de la marisma, probablemente debidas a flexiones del terreno, ya que en todas ellas existe un talud patente. situándose las descargas por debajo de los 10 m.s.n.m.

Las mayores fluctuaciones de los niveles freáticos parecen estar relacionadas con las descargas de aguas del acuífero regional. Esto podría relacionarse con lo observado a otra escala en el Corral Largo (MUÑOZ REINOSO 1997), es decir, cuanto mayor es la disponibilidad de agua (descargas del acuífero regional) mayores son las salidas (fluctuaciones). Las descargas más constantes permiten el desarrollo de formaciones vegetales con mayores requerimientos hídricos. La presencia de amplias bandas de helechos parece indicar en el área de Doñana la conexión con flujos de aguas subterráneas que deben mantenerse hasta cierto punto constantes, lo cual asegura el aporte de agua durante su ciclo de vida (primavera-verano). Los helechos no existen en la zona de dunas por la rnovilidad del sustrato ni por la irnportnncia de las descargas, siendo su presencia escasa en Ojillo y Acebuche.

A pesar de que el análisis se ha realizado durante una época de déficit hídrico, de recoger un número escaso de especies y pocos muestreos. se puede ensayar una tipificación de los ecosistemas de descarga sobre arenas de la Reserva Biológica de Doñana. que queda sintetizada en la Tabla 3. según las descargas predominantes, la fisicoquímica del agua subterránea. su vegetación y su morfometría y localización.

Esta tipificación representa un avance sobre las anteriores ya que incluye un análisis más global de las características de los ecosistemas acuáticos aún sin considerar su limnología. A pesar de incluir un reducido número de muestras (11), estas recogen una importante variabilidad de los ambientes acuáticos de la Reserva Biológica. No obstante, esta tipificación es mejorable y debe ser completada con nuevos criterios y datos, y debe ser contrastada con nuevos puntos de muestreo. Además. existen "lagunas" espaciales en el análisis, corno son las lagunas presentes en la zona central de la Reserva, no relacionadas en principio con complejos dunares (naves, dunas móviles) o zonas de descarga regional (vera).

Esta clasificación es, a grandes rasgos. coincidente con la de GARCÍA NOVO et al. (1991) en cuanto a la existencia de tres grandes zonas de descarga en la Reserva Biológica: contacto Naves-Manto Arrasado, Dunas móviles-Manto Arrasado y Manto Arrasado-Vera. En cambio, en la tipificación de estos autores en base a la evolución de la masa de agua libre, se interpreta que todas las lagunas con aguas poco mineralizadas se encuentran alimentadas por aguas superficiales (escorrentía o precipitación), dependiendo su evolución posterior a la naturaleza del sedimento.

En este trabajo se ha demostrado que las aguas que subyacen y alimentan las lagunas, a pesar de su baja rnineralización, presentan regímenes hídricos y composiciones químicas distintas (a los que se asocian comunidades vegetales distintas), lo cual depende de las descargas de aguas subterráneas predorninantes. Estas descargas han permitido distinguir cinco tipos de ecosistemas aciiáticos que se presentan en la Tabla 3 y se denominan utilizando la terminología utilizada en trabajos anteriormente citados.

Tabla 3. Clasificación de los ecosistemas acuáticos de la Reserva Biológica de Doñana. Conductividad en $\mu \mathrm{S} / \mathrm{cm}$.

Table 3. Doñana Biological Reserve aquatic ecosystems clasification. Electrical conductivity in $\mu \mathrm{S} / \mathrm{cm}$.

\begin{tabular}{|c|c|c|c|c|}
\hline $\begin{array}{l}\text { Ecosistemas } \\
\text { acuáticos }\end{array}$ & $\begin{array}{l}\text { Descargas } \\
\text { predominantes }\end{array}$ & Fisicoquímica & Vegetación & $\begin{array}{l}\text { Morfometría. } \\
\text { Localización }\end{array}$ \\
\hline $\begin{array}{l}\text { I permanenter } \\
\text { (Dulce S Olalla) }\end{array}$ & D regionales & $\begin{array}{l}\text { Cond }>500 \\
\text { Carbonat altos }\end{array}$ & 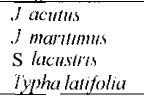 & $\begin{array}{l}\text { Tamaño medio-grande }>5 \\
\text { ha) } \\
\text { Profundas }(>1 \text { i m) } \\
\text { Peridutiares }\end{array}$ \\
\hline $\begin{array}{l}\text { I reniporctles } \\
\text { (Zahillo. Taraje) }\end{array}$ & $\begin{array}{l}\text { D regionales } \\
\text { D semilocales }\end{array}$ & $\begin{array}{l}\text { Cond } 500 \\
\text { Carbonat altos }\end{array}$ & $\begin{array}{l}\text { f. mantmins } \\
1 \text { actins } \\
\text { s holoschoems }\end{array}$ & $\begin{array}{l}\text { Tamaño medio }(* 5 \mathrm{ha}) \\
\text { Profundas }(>15 \mathrm{~m}) \\
\text { Peridunares }\end{array}$ \\
\hline $\begin{array}{l}\text { lefinueras } \\
\text { (Ojillo Acebuche) }\end{array}$ & D serrilocales & $\begin{array}{l}\text { Cond } 500 \\
\text { Paco mineralizadas }\end{array}$ & $\begin{array}{l}\mathrm{M} \text { negro } \\
\text { J effusss } \\
\text { I martmm }\end{array}$ & $\begin{array}{l}\text { Pequeñas ( }<1 \text { ha) } \\
\text { Someras } \\
\text { Manto Arrasado }\end{array}$ \\
\hline $\begin{array}{l}\text { Helechales } \\
\text { (Jahata M Pavón) }\end{array}$ & D regionales & $\begin{array}{l}\text { Cond }<500 \\
\text { Cl relativamente } \\
\text { aitos }\end{array}$ & $\begin{array}{l}\text { Paguilimm } \\
\text { 1 effusw } \\
\text { C longus }\end{array}$ & $\begin{array}{l}\text { Pequeñas ( } 1 \text { ha) } \\
\text { Someras } \\
\text { Vera }\end{array}$ \\
\hline $\begin{array}{l}\text { (orroles himedos } \\
\text { (C Largo) }\end{array}$ & D locales & $\begin{array}{l}\text { Cond }<200 \\
\text { Mineralización } \\
\text { escasa }\end{array}$ & S hotoschoems & $\begin{array}{l}\text { Encharcamiento periódico } \\
\text { Dunas moviles }\end{array}$ \\
\hline
\end{tabular}

\section{AGRADECIMIENTOS}

Parte de los muestreos conducentes a la realización de este trabajo han sido realizados dentro del marco del proyecto “Doñagua”. La Reserva Biológica de Doñana y el Parque Nacional de Doñana concedieron los permisos necesarios para la realización de los muestreos. En las tareas de campo colaboraron Poli, Yolanda. Dani y Agustín. Agradezco los comentarios realizados sobre la primera versión del manuscrito a los doctores García Novo, de Castro y Olías. 


\section{BIBLIOGRAFÍA}

ALLIER. C.F., F. GONZÁLEZ BERNÁLDEZ y L. RAMÍREZ DÍAZ 1974. Mapa Ecológico de la Reserva Biológica de Doñana. División de Ciencias del C.S.I.C. Estación Biológica de Doñana, Sevilla.

WORJA,F. y F. DÍAZ DEL OLMO 1987. Complejos húmedos del Abalario (Entornode Doñana, Huelva). Oxyura IV (1):27-41

BRAVO. M.A. y C. MONTES 1993. Inventario de las formaciones palustres del manto eólico del Parque Nacional de Doñana (SW España). Actas VI Congreso Español de Limnología, Granada: 31-43.

CUSTODIO, E. 1995. El papel de la Hidrología en los Programas de Restauración de Humedales en ambientes fluctuantes. En: Montes, C., G. Oliver, F. Molina y J. Cobos (eds). Bases ecológicas para la restauración de humedales en la Cuenca Mediterránea. Junta de Andalucia. pp. 43-60.

CUSTODIO, E., J. DOLZ. J. GUIMERÁ, M. MANZANO, R. PONCELA, J. SAMPER. M. SÁNCHEZ y E. VELASCO 1992. Aportaciones al conocimiento hidrogeológico de los acuífcros del Parque Nacional de Doñaria y su entorno. Hidrogeología y Recursos Hidráulicos XVI: 425-439.

FREEZE. R. A. \& J. A. CHERRY 1979. Groundwater. Prentice-Hall. New Jersey. 604 pp.

GARCÍA NOVO, F., D. GALINDO, J.A. GARCÍA SÁNCHEZ, C. GUISANDE, J. JÁUREGUI. T. LÓPEZ, N. MAZUELOS, J.C. MUÑOZ, L. SERRANO. J. TOJA 1991. Tipificación de los ecosistemas acuáticos sobre sustrato arenoso del Parque Nacional de Doñana. Actas 111 Simposio del Agua en Andalucía. Córdoba. pp. 165-176.

GILVEAR, D. J. \& R. J. McINNES 1995. Wctland hydrological vulnerability and the use of classification procedures: a Scottish case study. J. Env. Manag. 42: 403-414.

GONZÁLEZ BERNÁLDEZ, F., L. RAMÍREZ, A. TORRES y F. DÍAZ PINEDA 1977a. Estructura de la vegctación de marisma de la Reserva Biológica de Doñana (Huelva). I. Análisis factorial de datos cualitativos. Anales Edaf. y Agrob. (9-10): 989-1003.

GONZÁLEZ BERNÁLDEZ, F., L. RAMÍREZ, A. TORRES y F. DÍAZ PINEDA 1977b. Estructura de la vegetación de marisma de la Reserva Biológica de Doñana (Huelva). II. Estudio de un gradiente de salinidad. Anales Edaf. y Agrob. (9-10): 1005-1017.

GONZÁLEZ BERNÁLDEZ. F. 1988. Typology of wetlands and evaluation of the resources ihey represcnt. International Symposium on Hydrology of Wetlands in Semiarid and Aricl regions. Sevilla. pp. 7-36.
GONZÁLEZ BERNÁLDEZ, F.. P. HERRERA y C. LEVASSOR 1988. Groundwatcr indicator plants in the Madrid Aquifcr. International Symposium on Hydrology of Wetlands in Semiarid and Arid regions. Sevilla. pp. 37-39.

GONZÁLEZ BERNÁLDEZ, F. y C. MONTES (coord) 1989. Los humedales del acuifero de Madrid. Inventario y tipología basada en su origen y funcionamiento. Canal de Isabel II. Madrid. 92 pp.

IGME 1983. Hidrogeología del Parque Nacional de Doñana y su Entorno. Serv. Publ. Min. Industria y Energía. Madrid. $120 \mathrm{pp}+$ inapa.

LÓPEZ, T., J. TOJA \& N.A. GABALLONE 1991. Limnological comparison of two peridunar ponds in the Doñana National Park (Spain). Arch. Hydrobiol. 120: 357 378.

LÓPEZ, T., N. MAZUELOS AND J.C. MUÑOZ REINOSO 1994. Spatial and temporal variation in the ionic composition of shallow water table in Doñana National Park (SW Spain). Verh. Internat. Verein. Limnol. 25: 1438-1444.

LLAMAS, M.R. 1990. Geohydrology of the eolian sands of the Doñona National Park (Spain). Catena Supplement 18: 145-154.

MANZANO, M., E. CUSTODIO y R. PONCELA 1991. Contribución de la hidrogcoquímica al conocimiento de la hidrodinámica de los acuíferos en el área de Doñana. 111 Simposio del Agua en Andalucía. Córdoba. pp. 457-486.

MERINO, J., L. RAMÍREZ DÍAZ, F. SANCHO ROYO y A. TORRES MARTÍNEZ 1980a. Estudio ecológico de la vegetación de ecotono ("Vera") de la Reserva Biológica de Doñana (Huelva). I. Metodología y medio físico-químico. Anales Edaf. y Agmb. (11-12): 1867-1878.

MERINO, J., L. RAMÍREZ DÍAZ, F. SANCHO ROYO y A. TORRES MARTÍNEZ 1980b. Estudio ecológico de la vegetación de ecotono ("Vera") de la Reserva Biológica de Doñana (Huelva). II. Tipificación ecológica de las coinunidades. Anales Edaf. y Agrob. (11-12): 1879-1894.

MUÑOZ REINOSO, J.C. 1997. Patrón espacio-temporal del matorral de la Reserva Biológica de Doñana y sus relaciones con el Acuifero Almonte-Marismas. Tesis Doctoral. Univ. de Sevilla. 220 pp.+apéndices.

MUÑOZ REINOSO, J.C. 1995. Influencia del agua freática sobre la vegetación de las áreas de descarga sobre arenas de la Reserva Biológica de Doñana. Limnetica 11(2): 9-16.

RAMÍREZ DÍAZ, L. 1973. Estudio ecológico cuantitativo del matorral de la Reserva Biológica de Doñana. Tesis Doctoral. Universidad de Sevilla.

RODRÍGUEZ RAMÍREZ, A. 1996. Geomorfología continental y submarina del Golfo de Cádiz (Guadiana- 
Guadalquivir) durante el Cuaternario reciente. Tesis Doctoral. Univ. Huelva. $370 \mathrm{pp}$.

RODRÍGUEZ ARÉVALO, F.J. y M.R. LLAMAS 1986.

Evaluación preliminar del impacto de los boriibcos de agua subterránea en el ecotono de la Vera-Retuerta (Parque Nacional de Doñana). II Simposio sobre el Agua en Andalucía. Granada. 423-434.

SACKS. L. A., J. S. HERMAN, L. F. KONIKOW and A. L. VELA 1992. Seasonal dynainics of groundwatcr-lake interactions at Doñana National Park, Spain. J. Hydrology 136: 123-154.

SALVANY, J.M. 1991. Análisis de la estratigrafia y sedimentología de muestras de sondeos representativos en relación con el Parque Nacional de Doñana con vistas a la evaluación crítica de los modelos de flujo subterráneo en el acuifero. Asoc. Curso. Internal. Hidrología Subterránea, Barcelona. Informe para lo Comisión Internal. Expertos sobrc el Entorno de Doñana. Junta de Andalucía.
SALVANY. J.M. y E. CUSTODIO 1995. Características litoestratigráficas de los depósitos plio-cuaternarios del bajo Guadalquivir en el área de Doñana: implicaciones hidrogeológicas. Rev. Soc. Geol. España 8 (1-2): 21-31.

SERRANO, L. \& J. TOJA 1995. Limnological description ot four temporary ponds in the Doñana National Park (SW Spain). Arch. Hydrobiol. 133: 497-516.

SILJESTRÖM, P. 1985. Geomorfología y edafogénesis de las arenas clel Parque Nacional de Doñana. Tesis Doctoral. Univ. Sevilla. 515 pp.

ter BRAAK, C.J.F. 1991. CANOCO v. 3.12. Agricultural Mathematics Group. Wageningen.

VELA, A. 1984. Estudio preliminar de la hidrogeología e hidrogeoquímica del sistema de dunas móviles y flecha litoral clel Parque Nacional de Doñana. Tesis de Licenciatura. Universidad Complutense de Madrid. 